\title{
Use of the product of driving pressure and respiratory rate for predicting failure of weaning from mechanical ventilator in medical patients
}

Ju Gong ${ }^{127}$, Bibo Zhang ${ }^{37}$, Xiaowen Huang ${ }^{4}$, Bin $\mathrm{Li}^{5}$, Jian Huang ${ }^{6 *}$

${ }^{1}$ Department of Emergency medicine, The first affiliated Hospital of Soochow University, Suzhou,215006,ChinaＥmail:44707879@qq.com

${ }^{2}$ Department of Critical Care Medicine, The Affiliated Changshu Hospital of Xuzhou Medical University, Changshu,215500, China

${ }^{3}$ Department of Critical Care Medicine, The Affiliated Changshu Hospital of Xuzhou Medical University, Changshu,215500, China Email:zbb116@sina.com

${ }^{4}$ Department of acupuncture and tuina, Changshu Hospital of Traditional Chinese

Medicine, Changshu, 215500,China Email:tcmhuang@sina.com

${ }^{5}$ Department of Critical Care Medicine, The Affiliated Changshu Hospital of Xuzhou Medical University, Changshu,215500, China Email: $363394248 @ q q . c o m$

${ }^{6}$ Department of Emergency medicine, The first affiliated Hospital of Soochow University, Suzhou,215006, China

${ }^{7}$ These authors contributed equally to this work.

*Correspondence: manyiwu_1@163.com 


\begin{abstract}
Background: Respiratory workload increment in the process of mechanical ventilation withdrawal is critical for the determination of weaning outcome. Pressure, tidal volume $(\mathrm{Vt})$ and respiratory rate $(\mathrm{RR})$ are considered as patient's respiratory power, albeit being affected by excessive respiratory load. We aimed to evaluate the performance of driving pressure (DP) $\times \mathrm{RR}$ to predict the outcome of weaning.

Methods: Plateau pressure (Pplat) and positive end-expiratory pressure tot (PEEPtot) were measured during mechanical ventilation, viz., (1) brief deep sedation, (2) on volume support ventilation of MV with Vt $6 \mathrm{ml} / \mathrm{kg}$ and a PEEP of $0 \mathrm{~cm} \mathrm{H}_{2} \mathrm{O}$, (3) Pplat and PEEPtot were measured by holding breath for $2 \mathrm{~s}$ after inhalation and exhalation, respectively. The DP was determined as Pplat minus PEEPtot. The highest RR was recorded within 3 min during spontaneous-breathing trial (SBT). Patients that were able to tolerate SBT for $1 \mathrm{~h}$ were directly extubated.

Results: Out of the 61 patients studied, 22 failed weaning. During the withdrawal of ventilation, DP $\times$ RR was $134.2 \pm 33.2 \mathrm{cmH}_{2} \mathrm{O} \cdot$ breaths $/ \mathrm{min}$ and $238.5 \pm 61.7$ $\mathrm{cmH}_{2} \mathrm{O} \cdot$ breaths/min $(P=0.00)$, DP was $7.9 \pm 1.6 \mathrm{cmH}_{2} \mathrm{O}$ and $9.7 \pm 2.3 \mathrm{cmH}_{2} \mathrm{O}(P=0.00)$, in the "success" and "failure" groups, respectively. The $\mathrm{DP} \times \mathrm{RR}$ index greater than $170 \mathrm{cmH}_{2} \mathrm{O} \cdot$ breaths/min had a sensitivity of $95.5 \%$ and a specificity of $89.7 \%$, while DP index greater than $8.1 \mathrm{cmH}_{2} \mathrm{O}$ had $81.8 \%$ sensitivity and $64.1 \%$ specificity to predict weaning failure.
\end{abstract}

Conclusions: Measurement of DP $\times$ RR during withdrawal of ventilation may help predict weaning outcome. Noticeably, high $\mathrm{DP} \times \mathrm{RR}$ increased the likelihood of weaning failure.

Key Words: Driving pressure; mechanical ventilation; Ventilator weaning

\title{
1.Background
}

It has been shown that as soon as the factors causing the respiratory failure starts were removed, the weaning from mechanical ventilation should be initiated. However, even with the spontaneous-breathing trial (SBT) being used worldwide, more than $10 \%$ of extubations failed [1]. There are many weaning parameters, such as tracheal airway occlusion pressure at $0.1 \mathrm{~s}(\mathrm{P} 0.1)$ [2], rapid shallow breathing index (RSBI) [3], and CROP index (respiratory compliance, RR, oxygenation, maximum inspiratory pressure) [4]. Most importantly, RSBI seems to be the most substantial single parameter to predict weaning success with positive predictive value (PPV), negative predictive value (NPV), sensitivity and specificity of $0.86,0.82,0.87 \pm 0.14$ and $0.52 \pm 0.26$,respectively[3]. Nonetheless, this index had low specificity. At present, it is difficult for clinicians to choose the accurate timing of withdrawal of mechanical ventilation. In this study, we sought to identify a new parameter to improve accuracy and precision of weaning.

Airway driving pressure(DP) represents the cyclic strain which is related to volume change. Usually, this is a physiological way of adjusting Vt to the residual lung size (respiratory system compliance), there is a linear relationship between the decrease of compliance and the decrease of functional residual capacity. High DP impairs diaphragm function[5] apart from causing greater mortality[6-7].By extension, 
pressure applied to multiply volume change is the work that reflects respiratory workload, while affecting the outcome of weaning [8]. Thus, both DP and work are associated with weaning outcome. Currently, a number of observational studies have demonstrated an association between lung injury or mortality and mechanical power [9-10]. Pressure $\times$ Volume/Time=Power[11]. DP and work are important elements of mechanical power. Combined with unit time, power can reflect the respiratory load and endurance of breathing better. Thus, we postulated that $\mathrm{DP} \times$ respiratory rate $(\mathrm{RR})$ could be a predictor of weaning in patients with respiratory failure. In order to assess the predictive value (PV), we did an observational trial to investigate the utility of this index during weaning from mechanical ventilation (MV) to predict weaning outcomes.

\section{Methods}

This study was performed between March 2017 and June 2019 in the affiliated Changshu hospital of Xuzhou medical university. All the patients were recruited from the medical intensive care unit. The participants were enrolled into the study when they were ready for weaning from MV. Prior to the study, ethical approval for this study was obtained from the ethics committee of affiliated Changshu hospital of Xuzhou medical university (ID number: 0056-2017). All the patients or their relatives gave written informed consent prior to the investigation.

\subsection{Patient selection}

Inclusion criteria included age $\geq 18$ years, and MV for more than $24 \mathrm{~h}$. The readiness of the patients for weaning largely dependent on their ability to meet all the criteria: $\mathrm{PaO}_{2} / \mathrm{FIO}_{2}>200 \mathrm{mmHg}$, using PEEP $\leq 5 \mathrm{cmH}_{2} \mathrm{O}$ and fraction of inspired oxygen $\left(\mathrm{FIO}_{2}\right) \leq 0.4$, stable hemodynamic (heart rate $\leq 140$ beats $/ \mathrm{min}$, absence or lowdose vasopressors required i.e., dopamine or dobutamine $<5-10 \mathrm{ug} / \mathrm{kg}$.min), good consciousness (Glasgow coma scale $\geq 13$ ), stable metabolism (i.e., acceptable electrolyte levels), effectiveness of cough strength (positive white card test result), and in the recovery stage of disease.

\subsection{Exclusion Criteria}

Exclusion criteria included age less than 18 years, and MV for less than $24 \mathrm{~h}$, pregnancy, end-stage tumor, neuromuscular disease, presence of pneumothorax, flail chest, large pleural effusion, patients with severe deformity, obesity, organ failure before weaning, lack of informed consent.

\subsection{Study Design}

The patients who met the aforementioned inclusion criteria were injected intravenously with remifentanil $0.2 \mathrm{mg}$ for brief deep sedation. Next, when patients were awakened, they were put on triggering assisted breaths on volume support ventilation of MV with Vt $6 \mathrm{ml} / \mathrm{kg}$ of ideal body weight and a PEEP of $0 \mathrm{cmH}_{2} \mathrm{O}$. Respiratory mechanical parameters (Pplat and PEEPtot) were recorded. Pplat and PEEPtot were measured by holding breath for $2 \mathrm{~s}$ after inhalation and exhalation, respectively. The DP was measured as Pplat minus PEEPtot. The treating physician evaluated each case individually. When the study subjects were deemed ready for an SBT with the intent to weaning, each participant was disconnected from the ventilator to SBT for $1 \mathrm{~h}$ using $\mathrm{T}$ piece with oxygen supplementation $\left(\mathrm{FIO}_{2}\right.$ of $\left.0.25-0.5\right)$ to 
achieve pulse oxygen saturation $\left(\mathrm{SpO}_{2}\right)>90 \%$. Then, attending physicians who were not involved in this study recorded the maximal RR by ECG monitor during the 3-min SBT. After that, the DP multiplies by RR was calculated by investigator.

Notably, patients who passed the $1 \mathrm{~h}$ SBT were directly extubated, while others were reconnected to the ventilator with the previous ventilatory parameters if failed to the SBT. The decision to continue with the SBT, use noninvasive ventilation or reintubate after extubation was left up to the discretion of attending physicians who were not allowed to know the research indicators.

Intolerance signs: (1)RR $>35$ or $<8$ breaths/min; (2) Heart rate $<140$ beats/min or a 20\% increase or decrease from baseline; (3) SBP $>160 \mathrm{mmHg}$ or $<90$ $\mathrm{mmHg}$; (4)Arterial blood oxygen saturation $>90 \%$; (5) Sweating, agitation, decreased level of consciousness.

\subsection{Weaning failure definition}

Weaning failure was defined as either the failure of SBT or the need for reintubation within $48 \mathrm{~h}$ following extubation[12]. Patients who failed the SBT, or required reintubation or noninvasive ventilation (NIV) within $48 \mathrm{~h}$ of discontinuation of MV were considered as having failed to wean.

\subsection{Statistical analysis}

Normally distributed continuous variables were expressed as means with standard deviations. The variables were compared with Student's t test for independent samples. Non-normally distributed continuous variables were compared via MannWhitney U test, while categorical variables were compared with the Chi-Square Test or correction of continuity. Receiver operating characteristic (ROC) curve analysis was performed to assess the capability of $\mathrm{DP} \times \mathrm{RR}$ and DP to predict patients who may succeed at weaning or fail. Statistical analysis was performed with Statistical package for social sciences (SPSS) 17.0 software (SPSS, Chicago, IL, USA). A $p$ values less than 0.05 were chosen to indicate the statistical significance.

\section{Results}

During the study period, 61 patients were recruited with 22 of them failing weaning. Fifteen (15) of 22 the patients failed to pass weaning after $1 \mathrm{~h} \mathrm{SBT}$. Also, 2 patients in the failure group were reintubated within $48 \mathrm{~h}$ of extubation. The remaining 5 patients were initiated on NIV within $48 \mathrm{~h}$ of extubation either preemptively or as rescue therapy. Importantly, 39 patients were weaned successfully (Fig 1). The mean age of the study cohort was 67.7 years, with 42 (68.9\%) being male. The characteristics of the patients are summarized in Table 1. The difference between the two groups regarding age, gender, and acute physiology as well as Chronic Health Evaluation II score were not statistically significant. The duration of ventilation and ICU length of stay were longer in the failure group $(P=0.01, P<$ $0.0001)$ (Table 1). The patients in the failure group had significantly higher DP $\times \mathrm{RR}$ $(P<0.0001)$, and DP $(P<0.0001)$ in comparison with the success group. Notably, the $\mathrm{DP} \times \mathrm{RR}$ was $238.5 \pm 61.7 \mathrm{cmH}_{2} \mathrm{O} \cdot$ breaths $/ \mathrm{min}$ and $134.2 \pm 33.2 \mathrm{cmH}_{2} \mathrm{O} \cdot$ breaths $/ \mathrm{min}(P$ $<0.0001)$ in the failure and success groups; while the DP was $9.7 \pm 2.3 \mathrm{cmH}_{2} \mathrm{O}$ and $7.9 \pm 1.6 \mathrm{cmH}_{2} \mathrm{O}(P<0.0001)$ in the failure and success groups, respectively. The mean DP $\times$ RR and DP values between the two patient groups were compared in Fig 2 . 
A DP $\times$ RR index greater than $170 \mathrm{cmH}_{2} \mathrm{O} \cdot$ breaths/min had a sensitivity of $95.5 \%$ and a specificity of $89.7 \%$ to determine weaning failure (area under the curve [AUC], 0.968). Likewise, a DP index greater than $8.1 \mathrm{cmH}_{2} \mathrm{O}$ showed $81.8 \%$ sensitivity and 64.1\% specificity (AUC,0.742) (Table 2, Fig 3).

\section{Discussion}

It was observed in this study that $\mathrm{DP} \times \mathrm{RR}$ index was greater in the patients who failed weaning comparable to those who were successfully extubated from MV. In contrast with the most accurate parameter RSBI [3], our index showed similar sensitivity and higher specificity. Even RSBI has limitations, including mean RSBI value less than 105 in the failure group [13].

Imbalance between respiratory energy load and muscle function contribute to failure of ventilation weaning. Notwithstanding, several mechanisms may be involved, namely, alteration in airway resistance, respiratory system compliance, intrinsic positive end-expiratory pressure, and increased additional work (mechanism or intubation) are associated with excessive respiratory load. The alternative contribution of each factor affects the energy load. Actually, pressure, Vt and RR are considered as patient's respiratory power which is affected by excessive respiratory load. In this study, patients received the same Vt. Thus, pressure $\times \mathrm{RR}$ could be referred to respiratory power of patients amidst being affected by excessive respiratory load.

DP is defined as the amount of cyclic parenchymal deformation imposed on ventilated and preserved lung units [14], which is most accessible at the bedside, and may serve as a marker of cyclic lung strain [15]. Mathematically, Vt/system compliance (C) is the DP, i.e. the applied pressure above PEEP to deliver the Vt[16] . Patients who failed to withdraw from the machine were more likely to have persistent lung opacities and cardiac insufficiency, characterized by high driving pressure and poor lung compliance.

On the other hand, it was found in this study that the DP $\times$ RR and DP index were significantly greater in the failure group compared with the success group. Pertinently, DP $\times$ RR had higher sensitivity and specificity than the DP to predict weaning failure. Noticeably, most of the patients who were ready to withdraw after meeting the standard had their high airway resistance being relieved. Thus, respiratory system compliance mainly affected the respiratory load. In the case of poor thoracic or pulmonary compliance, when the patient changed from mechanical ventilation to SBT, the elastic load and the driving pressure of the patient increased and the weaning failed. In addition, when the patient's primary disease did not improve, the respiratory load was high. Besides, during SBT, the patient's respiratory frequency accelerated, indicating that the respiratory endurance was not enough to balance the patient's respiratory load[17]. As a result, the patient could not maintain a stable breath, while the final result was also a failure to withdraw the machine. Weaning is a complex process involving transition from ventilatory support to spontaneous breathing. Any single parameters cannot guide the weaning well. A more global perspective which combines DP and RR does not only reflects respiratory system compliance, but also the capacity to perform work (energy) and the intensity of energy application (power). 
When the required pressure to produce the fixed volume is high, work rate is increased, or when RR is increased, work per unit time is high coupled with high power, indicating that the patient is more likely to fail to withdraw from mechanical ventilation.

In this study, some few limitations were identified. Different etiologies were studied together, which thereby increased the heterogeneity of the study. Despite this, the DP $\times$ RR was significantly greater in the failure group compared with the success group. There are also limitations of the study generalizability as this study did not include obese patients (a growing part of the population), patients with "organ failure" (many ICU patients), and was still a single center study. In addition, the SBT failure was included in the definition of machine withdrawal failure, thus the result was greatly affected by RR index in SBT. The observation of weaning outcomes does not mean that an intervention targeted at achieving that profile will accurately determine the timing of weaning.

\section{Conclusion}

The $\mathrm{DP} \times \mathrm{RR}$ index may serve as a novel method to predict the outcome of weaning. A greater $\mathrm{DP} \times \mathrm{RR}$ index is predictive of failed weaning. This combination provide a clue for physicians as to how they assess weaning readiness in critically ill patients. Because of the complexity of factors that determine the outcome of weaning, the $\mathrm{DP} \times \mathrm{RR}$ index serves as a good but may be an imperfect predictor. Therefore, further evidences are required to validate the finding.

\section{Abbreviations}

VT: Tidal volume; RR Respiratory rate; DP Driving pressure; Pplat Plateau pressure; PEEPtot Positive end-expiratory pressure tot; SBT: Spontaneous breathing test; RSBI Rapid shallow breathing index; PPV Positive predictive value; NPV negative predictive value; MV: Mechanical ventilation; FIO2 Inspired oxygen; $\mathrm{SpO} 2$ pulse oxygen saturation; NIV Noninvasive ventilation; ROC Receiver operating characteristic; AUC: Area under curve.

\section{Declarations}

\section{Ethics approval and consent to participate}

The study was approved by the institutional ethics committee of the hospital, The Affiliated Changshu Hospital of Xuzhou Medical University (20170056). All patients or relatives of the patients gave written informed consent prior to participation.

\section{Consent for publication}

Not applicable.

\section{Availability of data and materials}

The datasets used and/or analyzed during the current study are available from the corresponding author on reasonable request.

\section{Competing interests}


The authors declare that they have no competing interests.

\section{Funding}

This work has received funding from the Science and Education and Science and Technology Project of Suzhou City(KJXW2017064). The funding had no impact on the writing of the manuscript.

\section{Authors' contributions}

GJ wrote large parts of the manuscript and conceived the study design and data collection. ZB B participated in the study design. HX W performed statistical analyses. LB participated in literature search. HJ reviewed the manuscript. All authors interpreted the data, contributed to the intellectual content, reviewed the manuscript, and approved the final version.

\section{Acknowledgements}

We thank all critical care fellows and nurses for their clinical assistance. We also thank the experts at BMC Pulmonary Medicine for reviewing our manuscript.

\section{References}

1. Ruan S-Y, Teng N-C, Wu H-D, et al. Durability of weaning success for liberation from invasive mechanical ventilation: an analysis of a nationwide database. Am J Respir Crit Care Med. 2017 Sep 15;196(6):792-795. doi:10.1164/rccm.2016102153LE.

2. Okamoto K, Sato T, Morioka T. Airway occlusion pressure (P0.1) - a useful predictor for the weaning outcome in patients with acute respiratory failure. $\mathrm{J}$ Anesth. 1990;4:95-101. doi: 10.1007/s0054000040095.

3. Tobin MJ, Jubran A. Variable Performance of Weaning-Predictor Tests: Role of Bayes' Theorem and Spectrum and Test-Referral Bias. Intensive Care Med. 2006 Dec;32(12):2002-2012. doi: 10.1007/s00134-006-0439-4.

4. El-Khatib MF, Bou-Khalil P. Clinical review: liberation from mechanical ventilation. Crit Care. 2008;12:221.doi: 10.1186/cc6959. Epub 2008 Aug 6.

5. Goligher EC, Fan E, Herridge MS, et al. Evolution of Diaphragm Thickness during Mechanical Ventilation. Impact of Inspiratory Effort. Am J Respir Crit Care Med. 2015;192(9):1080-1088. doi:10.1164/rccm.201503-0620OC.

6. Amato MB, Meade MO, Slutsky AS, et al: Driving pressure and survival in the acute respiratory distress syndrome. N Engl J Med.2015;372:747-755.doi: 10.1056/NEJMsa1410639.

7. Aoyama H, Pettenuzzo T, Aoyama K, et al. Association of Driving Pressure With Mortality Among Ventilated Patients With Acute Respiratory Distress Syndrome: A Systematic Review and Meta-Analysis. Crit Care Med. 2018 46(2):300-306.doi: 10.1097/CCM.0000000000002838.

8. Fiastro JF, Habib MP, Shon BY, et al. Comparison of standard weaning parameters and the mechanical work of breathing in mechanically ventilated 
patients. Chest. 1988;94(2):232-238. doi:10.1378/chest.94.2.232

9. Cressoni M, Gotti M, Chiurazzi C, et al. Mechanical Power and Development of Ventilator-induced Lung Injury.Anesthesiology. 2016 May;124(5):1100-8. doi: 10.1097/ALN.0000000000001056.

10. Zhang Z, Zheng B, Liu N, et al. Mechanical power normalized to predicted body weight as a predictor of mortality in patients with acute respiratory distress syndrome. Intensive Care Med.2019 45(6):856-864.doi: 10.1007/s00134-01905627-9.

11. Marini JJ.Dissipation of energy during the respiratory cycle: conditional importance of ergotrauma to structural lung damage. CurrOpinCrit Care. 2018 Feb; 24(1):16-22. doi: 10.1097/MCC.0000000000000470.

12. Boles JM, Bion J, Connors A, et al. Weaning from mechanical ventilation. Eur Respir J. 2007;29(5):1033-1056.doi: 10.1183/09031936.00010206.

13. Palkar A, Narasimhan M, Greenberg H, et al. Diaphragm Excursion-Time Index A New Parameter Using Ultrasonography to Predict Extubation Outcome. CHEST. 2018;153(5):1213-20.doi: 10.1016/j.chest.2018.01.007. Epub 2018 Jan 17.

14. Amato MBP, Meade MO, Slutsky AS, et al. Driving pressure and survival in the acute respiratory distress syndrome. N. Engl. J. Med. 2015; 372: 747-55.doi: 10.1056/NEJMsa1410639.

15. Protti A, Andreis DT, Monti M, et al. Lung stress and strain during mechanical ventilation: any difference between statics and dynamics? Crit. Care Med. 2013; 41: 1046-55.doi: 10.1097/CCM.0b013e31827417a6.

16. MacIntyre N. Ventilator Management Guided by Driving Pressure: A Better Way to Protect the Lungs? Crit Care Med.2018 46(2):338-339.doi: 10.1097/ CCM.0000000000002882.

17. Hart N, Hawkins $\mathrm{P}$, Hamnegård $\mathrm{CH}$, et al. A novel clinical test of respirat ory muscle endurance. Eur Respir J. 2002;19(2):232-239. doi:10.1183/090319 36.02.00247602.

\section{Figure Legends}

Fig.1 Flowchart of the study. $D P$ (driving pressure); $R R$ (respiratory rate); $S B T$ (spontaneous breathing trial). Fig.2 DP $\times$ RR,DP: Comparison of two patient group.

Fig.3 Receiver operating characteristic curve for $\mathrm{DP} \times \mathrm{RR}$ and DP. The $\mathrm{DP} \times \mathrm{RR}$ :AUC 0.968(95\%CI 0.91-1.00 $P<0.0001$ ),DP: AUC 0.742(95\%CI 0.61-0.88 $P<0.0001$ )

\section{Table Legends}

Table.1 Patient Demographics. APACHE=Acute Physiology and Chronic Health Evaluation; LOV=length of ventilation; LOS =length of stay; ${ }^{\mathrm{a} C o m p a r e d}$ with continuity corrections for the Chi-Squared Test. Table.2 Comparison Between Diagnostic Tests. $\mathrm{DP}=$ driving pressure; $\mathrm{RR}=$ respiratory rate 